\title{
RETIRO LABORAL Y AJUSTE A LA JUBILACIÓN DE HOMBRES Y MUJERES EN LA MEDIANA EDAD
}

WORK RETIREMENT AND ADJUSTMENT TO RETIREMENT OF MEN AND WOMEN IN THE MIDDLE-AGED

Volumen 11, Número 1

pp. $1-28$

Este número se publicó el 30 de abril de 2011

Roxana Cruz Meléndez

La revista está indexada en los directorios:

LATINDEX, REDALYC, IRESIE, CLASE, DIALNET, DOAJ, E-REVIST@S,

La revista está incluida en los sitios:

REDIE, RINACE, OEI, MAESTROTECA, PREAL, HUASCARAN, CLASCO 


\title{
RETIRO LABORAL Y AJUSTE A LA JUBILACIÓN DE HOMBRES Y MUJERES EN LA MEDIANA EDAD WORK RETIREMENT AND ADJUSTMENT TO RETIREMENT OF MEN AND WOMEN IN THE MIDDLE-AGED
}

\section{Roxana Cruz Meléndez ${ }^{1}$}

\begin{abstract}
Resumen: En este artículo se presentan los resultados de una investigación aplicada cuyo objeto de estudio fue un grupo de hombres exfuncionarios y mujeres exfuncionarias del área administrativa de la Universidad de Costa Rica (UCR) que se encuentran en la etapa de vida denominada "la mediana edad" y que enfrentan el proceso trabajo-retiro laboral. El estudio consistió en identificar los principales factores que inciden en el retiro laboral a una edad más temprana; la viviencia del proceso, el ajuste a la jubilación y sus implicaciones a nivel personal y social. La información recopilada se obtuvo a partir de la técnica "la entrevista fenomenológica" concebida como una exploración profunda que proporciona datos de gran valor conceptual y humana; en ella, tanto la persona que entrevista como la entrevistada mantienen un compromiso para que la búsqueda de la información sea dentro de una relación empática. Partiendo de lo subjetivo por su historia de vida al mandato social por lo roles asignados y asumidos por la población en estudio. Los resultados obtenidos indican que pareciera que la valoración que cada uno de los participantes hace de su rol laboral y el significado del trabajo en sus vidas es determinante en el proceso de retiro laboral y su ajuste al rol de persona jubilada.
\end{abstract}

Palabras clave: GÉNERO, TRABAJO, RETIRO LABORAL, JUBILACIÓN, MEDIANA EDAD.

Abstract: This article presents the results of applied research study that was aimed at a group of men and women, former administrative staff of the University of Costa Rica that are in the stage of life called middle age and facing work-retirement process work. The study consist in identify the main factors affecting the removal from work at a younger age, the experiencing process, adjustment to retirement and its implications for personal and social level. Information collected was obtained from the technique "the phenomenological interview "conceived as a deep exploration that provides valuable data conceptual and human, in her interview both the person and the respondent maintained a commitment to seeking information is within an empathic relationship. Based on the subject for his life story as mandated social roles assigned and assumed by the study population. Thus it seems that the valuation that each participant makes their job role and meaning of work in their lives is crucial in the process of withdrawal labor and adjusting to the role of retiree.

Key words: GENDER, WORK, RETIREMENT JOB, RETIREMENT, MIDDLE-AGED.

\footnotetext{
${ }^{1}$ Magister en Orientación de la Universidad de Costa Rica. Profesora en la Escuela de Orientación Educativa de la Universidad Católica de Costa Rica.
}

Dirección electrónica: roxanna1957@yahoo.es

Artículo recibido: 2 de junio, 2010

Aprobado: 28 de marzo, 2011 


\section{INTRODUCCIÓN}

En nuestra sociedad, el trabajo y la condición de género determinan la vida del ser humano; hombres y mujeres están condicionados a desempeñar funciones, actividades y comportamientos, básicamente por sus características sexuales, características que se van conformando a lo largo de la vida, y que el ámbito laboral complementa con la asunción de roles en torno a la posesión o carencia de cualidades físicas, intelectuales y emocionales.

El trabajo y las características que justifican su especialización es dialéctica en la relación hombre-mujer frente al trabajo. Ambos se definen y son diferentes al asumir las características que los hace ser hombres y mujeres pertenecientes a géneros distintos por su definición frente al trabajo (Lagarde, 1996).

En nuestra sociedad, ser hombre está asociado a la productividad de riquezas, a lo creativo, al trabajo socialmente útil y al poder económico. Tradicionalmente, el varón ve en el trabajo no solo la independencia económica, sino el medio de mantener su hegemonía que contribuye, a su vez, en su realización personal y social.

Como el trabajo sólo se ha concebido como una actividad que implica una remuneración, a la mujer no se le valora la contribución histórica, como reproductora y productora, aunque en el rol de madres y esposas, las mujeres han producido y reproducido la mano de obra para mantener en parte la estabilidad económica del hogar y de la sociedad.

No es sino con la incursión de la mujer en el mercado laboral que se producen cambios respecto al papel de la mujer en la economía, factor que contribuye en la búsqueda de la equidad de los diferentes roles que desempeñan las mujeres, tanto en su condición de madre y esposa como de trabajadora, de modo que al igual que el hombre, la mujer visualiza en el trabajo remunerado la independencia económica y su realización personal.

Por el desarrollo humano y las Garantías Sociales, la expectativa y calidad de vida de los y las costarricenses ha aumentado. Asimismo, los avances científicos y tecnológicos favorecen las condiciones laborales que inciden en la capacidad y en el desempeño laboral de hombres y mujeres, a edades que antes se consideraban como personas viejas, por lo que una persona trabajadora que se encuentra en la denominada etapa del adulto mayor es productiva, y ha adquirido el máximo de destrezas, conocimientos y habilidades, siendo un ente transmisor de esos conocimientos, lo que resulta muy valorado en las organizaciones. 
Por la valoración social que tiene el trabajo remunerado en la vida de hombres y mujeres, el cese laboral influye en la vida del trabajador, su familia y su entorno, ya que se rompe con lo establecido y conocido para enfrentar un nuevo estilo de vida, donde su rol laboral ya no es el condicionante que rige su vida.

En Costa Rica, a causa de los cambios y reformas en la legislación sobre pensiones, especialmente en el régimen de Pensiones y Jubilaciones del Magisterio Nacional, se ha incrementado, en los últimos años, el número de personas que se encuentran en la etapa de la mediana edad, periodo que abarca un rango de edad entre los 40 y los 65 años y que se acogen a la pensión y a la Jubilación.

Ante estos cambios, se ha presentado un fenómeno de creciente importancia, sobre todo, con respecto al personal de la Universidad de Costa Rica (UCR), el retiro laboral en una etapa de vida más temprana.

Con el fin de profundizar en este fenómeno e identificar tanto las repercusiones como las necesidades de orientación de un grupo de hombres y mujeres en la mediana edad, pensionados de la Universidad de Costa Rica, se propone responder las siguientes interrogantes:

¿Cómo incide la condición de género en la transición y ajuste al proceso trabajo-retiro laboral en un grupo de exfuncionarios y exfuncionarias administrativas de la Universidad de Costa Rica que se encuentran en la mediana edad?

¿Qué los motiva al retiro laboral?

¿Cómo asumen el proceso de trabajo-retiro laboral?

¿Cómo se visualizan en su condición de jubilados?

¿Cuáles ajustes personales y sociales enfrentan durante este proceso?

Para responder a estas preguntas, se realizó un acercamiento cualitativo que permitió profundizar en el significado que las personas dan a sus vivencias.

\section{ACERCAMIENTO TEÓRICO}

\subsection{Caracterización de la persona adulta en la etapa de la vida media}

La adultez es una condición social, biológica, orgánica y emocional. El ser humano no puede asumirse como adulto si no se reconoce como hombre o mujer en esa etapa de vida.

Desde el punto de vista teórico, la adultez implica madurez emocional, un alto grado de estabilidad emocional, un buen control de los impulsos y una elevada tolerancia a la Volumen 11, Número 1, Año 2011, ISSN 1409-4703 
frustración. En esta etapa se da la crisis de la mitad de la vida, que se caracteriza por el enfrentamiento a una serie de retos y tareas, que consisten en replantear sus metas e intereses para prepararse para la siguiente etapa de la vida.

Muchos hombres y mujeres adultas experimentan desilusiones con respecto a su trabajo, ya que no lo encuentran interesante. Se enfrentan con que la dependencia de los hijos con respecto a ellos como padres y madres disminuye.

Al respecto, Swensen (1983) y Goldstine (1977) citados por Rice (1997) señalan que en cuanto a la vida de pareja en la adultez, las necesidades matrimoniales dependerán de cómo lo hayan experimentado a lo largo de los años, es común que la satisfacción matrimonial disminuya, por lo que una pareja, cuya descendencia es independiente, ahora se tienen sólo uno al otro y para enfrentar la tarea de resolver los problemas y resentimientos para acercarse una vez más. Otra de las tareas propias del desarrollo en esta etapa consiste en la responsabilidad de brindar apoyo a sus padres que envejecen.

De acuerdo con Rice (1997) la mediana edad trae un cambio en el foco de las actividades sociales, ahora se siente una mayor necesidad de participar en actividades sociales, la madurez trae consigo un mayor interés en la diversión, en atender los propios intereses y pasatiempos o bien en desarrollar nuevas actividades.

Cuando estas tareas son resueltas adecuadamente se considera como un período de crecimiento y satisfacción personal, de lo contrario, puede ser considerado como un periodo de estancamiento, tal y como lo plantea Erikson citado por Rice (1997), la mediana edad puede ser un tiempo de expectativas propias de la condición de género no cumplidas, pero también se puede desarrollar una conciencia más positiva de nuevas expectativas y sueños.

\subsection{Condición de Género}

Nacer como mujer o como hombre, parece ser razón suficiente para heredar roles, funciones y patrones de comportamiento que conllevan un estilo de vida masculino o femenino. La socialización es el elemento clave para adquirir la identidad de género en el ser humano.

En la cultura patriarcal ser hombre se asocia con la productividad de riquezas, a lo creativo, al trabajo socialmente útil, al poder económico, a la capacidad, al buen desempeño y, sobre todo, a la competitividad que genera el mercado de trabajo que demanda precisión, creatividad y triunfó (Fernández, 2000). 
En el patriarcado, la fuente principal de la identidad masculina está relacionada con la actividad ocupacional. La actividad regular del hombre es el quehacer profesional, de ahí que se considere como una fuente de identidad la función proveedora y protectora que realiza el hombre Gomáriz (1997).

Mientras tanto, Marcela Lagarde (1992) señala que el poder masculino se caracteriza por la posibilidad política de ser hegemónicos, de permitir el dominio sobre los otros y la dirección del mundo. Las sociedades patriarcales son sociedades de trabajo, de hegemonismo y poder, y tener la posibilidad de existir depende de realizar dichas funciones. Ser hombre adulto significa ejercer pequeños poderes domésticos y familiares, así como tener sobre quién ejercer el poder, y ser padre significa, desde el patriarcado, ser tutor de otros, proveedor de bienes y estatus.

Por su parte, la formación de la identidad femenina es un proceso construido desde la infancia a partir de los aprendizajes en la familia. En este proceso, la mujer interioriza que para ser mujer debe cumplir con los roles impuestos socialmente de madre y esposa principalmente. "Históricamente, los roles sociales de las mujeres se han solapado y no ha habido necesidad funcional de distinguir entre tipos de roles sexuales. La expectativa era que las mujeres reales normales se convertían en esposas y madres", (Fernández, 1998, p.147).

Dentro del modelo capitalista-patriarcal a las mujeres se les asignan las tareas productivas y reproductivas, cumpliendo con el legado social de cuido y crianza de la población. Cumplir con este legado le ha dado a la mujer un lugar especial y prioritario en la sociedad, pero, al mismo tiempo, la ha esclavizado y la pone en una situación de vulnerabilidad y discriminación social (Villarreal, 2005).

El fenómeno de las mujeres trabajando en lo público no acaba de ser asimilado, pues la mujer se ve en una contradicción entre el goce y el sufrimiento que produce el trabajo pues tienen, por un lado, la vida doméstica y, por el otro, la vida pública y no logran estructurar una identidad integrada, lo que Lagarde ha llamado "la doble vida" (Lagarde, 1992).

Es así como la mujer vive cada día valores dobles, lenguajes distintos, exigencias diferentes y derechos a veces antagónicos. Algunos autores han llamado a esta paradoja la esquizofrenia vital de las mujeres, no en el sentido patológico, sino en el sentido de la separación que se da en una identidad que no acaba de conformarse. 
A pesar de esto, hoy se encuentran mujeres en aquellos espacios donde antes solo había hombres. En América Latina es manifiesta la presencia de mujeres en muchos espacios tradicionalmente masculinos "y esa vivencia de ser las primeras que estudiaron, hicieron algún oficio u ocuparon posiciones de poder político es para ellas un hecho muy importante" (Lagarde, 1992, p. 45).

Siguiendo la misma línea, para las mujeres, desplazarse del territorio doméstico a los territorios públicos significa sumar actividades, territorio y lenguajes, pero esto tiene un alto precio, las dobles y hasta triples jornadas. Sobre todo porque se da una contradicción en la autoidentidad de las mujeres, quienes se conciben "Ser para los otros frente a Ser para mî". En el caso de las mujeres, el Ser para los otros permite una autoidentidad de seres cuyo Yo se realiza en los otros.

\subsection{Significado del trabajo.}

El concepto de trabajo se define como una secuencia cronológica de todas las experiencias relacionadas con él, siendo el resultado de los estudios previos, de las prácticas pre laborales, los aprendizajes y el trabajo en sí mismo (Hoyt citado por Rodríguez, 1992).

Desde el punto de vista psicosocial el trabajo cumple una función integrativa o significativa al dar sentido a la vida en la medida en que le permite a la persona trabajadora realizarse; es, por lo tanto, una fuente de autoestima sana.

De igual forma, el trabajo mantiene a la persona trabajadora bajo una actividad más o menos obligatoria, proporciona un marco de referencia de una actividad regular, necesaria y con un propósito, es así como las personas están obligadas a trabajar si quieren mantener otras funciones vitales. Sin embargo, el significado personal del trabajo está en función de las experiencias vividas, significado que se configura, modifica y reafirma constantemente.

Como lo señala Burin y Meler (2001) para la mujer, la experiencia de adquirir poder, que generalmente acompaña el hecho de generar recursos, es lo que sostiene la tendencia actual de prepararse para el trabajo y a subjetivarse como mujeres trabajadoras.

Para Murillo, el trabajo en las mujeres tiene un significado diferente, ya que existe una relación especial que se da entre el espacio público y la esfera doméstica, pues los requerimientos del entorno laboral son dispares con las demandas del hogar, el solo hecho de las cargas familiares y las expectativas de tener hijos limita seriamente las oportunidades de entrada y continuidad en el empleo (Murillo, 1996). 
Sin embargo, la incorporación de la mujer al mercado laboral no solo implica la reivindicación de sus derechos, sino la oportunidad de demostrar su capacidad física e intelectual (Marín y otros, 1984).

El proceso de reivindicación de los derechos de las mujeres, su creciente participación en la vida pública, la reducción del Estado y la restricción de sus inversiones hace que, sin eximirlas de la carga del trabajo de la reproducción social, deban aumentar su aporte remunerado al interior del hogar Hermida (2006).

\subsection{Transición trabajo-retiro laboral}

La Teoría del Desarrollo de Carrera señala que el término transición puede estar referido a crisis, transformación o cambio. La transición no es tanto un cambio, sino la percepción de que se produce un cambio.

En este caso, la transición trabajo retiro laboral, puede ser percibido como un cambio en su rol de persona trabajadora o productiva hacia un rol más pasivo o menos productivo, (Rodríguez, 1992).

La transición se enmarca en el proceso de desarrollo del individuo, en su ciclo vital y se concibe como un paso de movilidad entre roles que exigen cambios de tipo personal y social permitiendo su desarrollo integral como un ser psicosocial.

En la etapa de vida de la población en estudio, se espera que la transición que enfrenten sea la transición trabajo-trabajo que conduce a la plena inserción laboral y se traduce en una serie de actividades e iniciativas que buscan la realización profesional. Sin embargo, en este proceso de realización profesional puede darse la crisis a mitad de carrera que conlleva a una reflexión y se concreta en diferentes situaciones como el abandono de la profesión u ocupación, cambio de empresa o trabajo, el reciclaje, la nueva formación, así como periodos de empleo y desempleo.

Además, la transición del trabajo al retiro profesional implica que la persona trabajadora se dispone a vivir otro tipo de vida adulta. El ocio va a ocupar la mayor parte del tiempo, siendo cada vez más protagónico en el desarrollo de la carrera del individuo y para afrontarlo se debe tomar en cuenta la dimensión personal, los sentimientos, la realidad, la madurez personal, las actitudes y motivaciones, así como la dimensión situacional, en fin la realidad socio-laboral en que este inserto. 


\subsection{Ajuste y adaptación a la jubilación}

El hombre y la mujer tienen que enfrenarse a diferentes situaciones y medios como parte de su propio desarrollo. Deben utilizar todos sus recursos internos y externos para lograr su adaptación, sobre todo si se considera la adaptación como la modificación de las condiciones de vida para ponerse en armonía con un medio diferente al vivido hasta entonces.

La adaptación del rol de persona trabajadora al rol de persona jubilada implica cambios de las fortalezas y recursos personales. Desde el punto de vista de la transición trabajo-retiro laboral y desde la jubilación, período en el cual la persona se enfrenta a cambios físicos propios de la edad y el prejuicio social asociado al envejecimiento, los hombres y mujeres deben recurrir a los factores internos y externos para lograr una mejor adaptación.

De acuerdo con Mata, algunos de los factores que intervienen en el ajuste de la persona trabajadora a la jubilación están en función del reconocimiento sobre la diferencia entre pensión y jubilación, la capacidad para tomar decisiones, y de las implicaciones reales de la jubilación, así como de la capacidad para identificar la crisis del desarrollo, dado que puede ver amenazada su identidad al sufrir cambios en su rutina diaria, en sus roles sociales, en el tipo de actividad que realiza y en otros aspectos que pueden generar una crisis (Mata, 2001)

Igualmente, señala que la capacidad para explorar nuevos intereses vocacionales es otro factor que contribuye al ajuste. "En alguna medida la persona echa mano a este banco de intereses en el momento que se jubila con el fin de ofrecerse a si misma la oportunidad de realizar actividades que le brinden calidad a su vida" (Mata, 2001, p. 31).

De manera que para un mejor ajuste en el proceso resulta de gran utilidad reconocer las pérdidas asociadas con la jubilación y entender la necesidad de perder o dejar atrás algo para crecer.

\subsection{Jubilación}

La jubilación se considera como un evento inevitable de la vida y se define como un cambio que se da al final del ciclo de desarrollo de la persona y funciona como un ritual que marca la transición entre la etapa adulta y la vejez. En la mayoría de los casos, coincide la jubilación con el inicio de los cambios físicos, psicológicos y sociales asociados a la etapa de la vejez (Papalia citado por Mora, 1997). 
De igual manera, la jubilación no es el cese definitivo de toda actividad sino el retiro del trabajo, y afecta las relaciones sociales y familiares de la persona jubilada. La pérdida del rol social y laboral conlleva un proceso de duelo que depende de la forma en que cada persona la experimente. Este sentimiento de pérdida es reforzado por la idea generalizada socialmente sobre la importancia del trabajo y el sentimiento de utilidad que se le da a la persona.

Se asocia la jubilación a la pérdida del rol social y laboral, de compañeros de trabajo por lo que ante estas pérdidas la persona pasa por un proceso de duelo y la forma en que cada persona la experimente es única y dependerá de las diferencias de personalidad, culturales y religiosas entre otras. (Amén y Fonseca, 1977, p. 13)

Según Alpízar, (1994) citado por Amén (2002) el proceso de jubilación incluye algunos aspectos como:

\section{Fase de euforia.}

2. Fase de ocupación.

3. Fase de la luna de miel.

4. Fase de desencanto.

5. Fase de la reorientación.

6. Fase de la estabilidad.

Sin embargo, la jubilación está condicionada por el significado del rol de trabajo y la condición de género, así por ejemplo si para la persona trabajadora, tanto hombres como mujeres, el rol laboral fue fundamental en su vida, la pérdida derivadas de la jubilación van a ser muy importantes, de lo contrario no representan una pérdida importante. A partir de la jubilación se da, por lo tanto, una resignificación de los roles desempeñados hasta el momento.

\section{ACERCAMIENTO METODOLÓGICO}

\subsection{Tipo de Estudio}

El estudio parte del paradigma de investigación naturalista utilizando el método fenomenológico. De acuerdo con Hernández y otros (2004) en este paradigma las preguntas surgen como parte del proceso de investigación y se caracteriza por ser flexible, moverse entre los eventos, su interpretación, las respuestas y el desarrollo de la teoría; además, tiene Volumen 11, Número 1, Año 2011, ISSN 1409-4703 
un común denominador: el patrón cultural, que parte de la premisa de que toda cultura 0 sistema social tiene su forma de entender las cosas y eventos, en este caso, la transición trabajo-retiro laboral.

Por su parte, el método fenomenológico, tal y como lo señala Dilthey citado por Mardones (1994), justifica la existencia de las ciencias humanas o de espíritu con el hecho de que en estas ciencias el objeto de estudio es el mundo del ser humano, producto de su espíritu y creado históricamente por este mismo, por lo que no se puede desvincular la persona investigadora de la realidad investigada, lo que permite reconstruir las percepciones, concepciones y experiencias del proceso de transición trabajo-retiro laboral y ajuste a la jubilación de las personas participantes.

Por eso, para reconstruir el proceso de transición trabajo-rol laboral y sus implicaciones, es necesario entender y conocer los modelos culturales en los que está inmersa la población, como el caso de los exfuncionarios y exfuncionarias de la Universidad de Costa Rica, que fueron objeto de estudio.

Desde el punto de vista del proceso de investigación, la fenomenología permitió conocer la influencia que tiene la condición de género y la etapa de vida en el proceso trabajo-retiro laboral de un grupo de exfuncionarias y exfuncionarios administrativos de la Universidad de Costa Rica. En tal sentido, lo importante es comprender lo concebido y subjetivo de las personas funcionarias universitarias que enfrentan un hecho dado históricamente, como el retiro laboral en una etapa de vida que conlleva, en muchos casos, un replanteamiento existencial.

Para conocer las percepciones, concepciones y experiencias, se seleccionó la entrevista fenoménica que permitió establecer una relación empática, profunda y de ayuda mutua entre el entrevistador y el entrevistado. De esta forma, para lograr los insumos necesarios en la realización de las entrevistas de definieron las siguientes áreas:

\section{Trabajo}

- Transición y ajuste.

- Jubilación.

Al ser una investigación cualitativa con un método fenomenológico se consideró realizar entrevistas a tres hombres y tres mujeres exfuncionarias de la Universidad de Costa Rica. 


\subsection{Caracterización de las personas participantes}

El proceso de selección de la población participante es esencial en la investigación cualitativa para asegurar un estrecho ajuste entre los datos y lo que la gente realmente hace y dice. Se hace una selección deliberada e intencional de acuerdo a los criterios y atributos establecidos (Rodríguez y colaboradores, 1996).

\subsection{Criterios de Participación}

Tomando en cuenta el interés de la investigadora, se eligieron como personas participantes a un grupo de seis exfuncionarios, tres hombres y tres mujeres del recinto "Ciudad Universitaria Rodrigo Facio", con edades entre los 45 y 59 años, que hubieran trabajado en la Universidad por espacio de, por lo menos, 10 años en el área administrativa, y que se acogieron a la pensión bajo las leyes 2248 y 7268 del Régimen de Pensiones del Magisterio Nacional.

Haber laborado por más de 10 años permite aportar información fundamental para entender determinados hechos y modos de comportamiento con respecto a este proceso de transición y ajuste a la jubilación. Además, se toma como atributo la edad, específicamente el rango que comprende la mediana edad, porque se considera que esta etapa es, por lo general, el período más fructífero y creativo del trabajo profesional, es una etapa en la que algunas personas, al darse cuenta que su trabajo ya no es tan interesante, pueden empezar a reconsiderar lo que desean hacer a futuro (Rice, 1997).

\subsection{Verificación de los resultados}

Para efectos de verificar los resultados obtenidos en la investigación se realizó una triangulación de la información obtenida. La triangulación suele ser concebida como un modo de protegerse de las tendencias del investigador, de confrontar y someter a control los relatos de los diferentes informantes, y se conceptúa como una estrategia para validar los resultados obtenidos con los métodos individuales (Taylor y Bogdan, 1998).

Además, permite contemplar los distintos elementos del estudio. Se basa en las fuentes de datos, la teoría, la metodología y el papel que desempeña la persona investigadora. De acuerdo con Denzín citado por Flick (2004) se pueden hacer cuatro tipos de triangulación, a saber: la triangulación de datos, la triangulación de quien investiga, la triangulación de la teoría y, por último, la triangulación metodológica. 
Para la presente investigación se utilizó la triangulación de datos, ésta se refiere a la utilización de diferentes fuentes de datos como el fundamento teórico, la información recopilada con los sujetos participantes y, por último, los datos de quien investigó.

\section{Análisis de Resultados.}

Con la información se dio respuesta a las preguntas planteadas en investigación, las cuales sirvieron de guía para la presentación de los resultados.

\section{1. ¿Qué induce a mujeres y hombres al retiro laboral?}

Para dar respuesta a esta interrogante se enmarcan los factores que inciden en tres categorías: el cambio de las condiciones laborales y legales, la posibilidad de dedicarse a otra actividad y, por último, los factores económicos.

El factor más influyente en la decisión del retiro laboral fue la posibilidad de perder los derechos y garantías labores, ante los cambios en la Ley de Pensiones del Magisterio Nacional.

"Se veía venir lo del plan fiscal, eso... que las prestaciones las iban a bajar y todas esas cosas y yo dije: jah no!, a mí no me van a agarrar con eso” (mujer No. 3).

Otro de los factores fue la percepción de los participantes de tener la oportunidad de realizar otras actividades; Sin embargo, llama la atención que en las mujeres este cambio de actividad sigue enfocado al aspecto laboral, pues consideraron la posibilidad de dedicarse a otras actividades fuera de su rol como madres y esposas, lo que evidenció la necesidad de mantener su lugar en el espacio público y no encasillarse, dentro de su condición de persona jubilada, en un rol de madres y esposas.

También, se notó en ellas resistencia a perder el espacio que con tanto sacrificio habían logrado, aunque siempre tuvieron el doble rol de madre-esposa y trabajadora, que concuerda con el posicionamiento de la Teoría del Desarrollo de Carrera, que indica que en la mediana edad puede darse la denominada crisis de mitad de la carrera y que lleva a un replanteamiento de las metas y expectativas actuales (Alvarez, 1995).

Por último, el factor económico incidió en la decisión de retiro al considera la posibilidad de obtener así estabilidad económica mediante la pensión. 


\section{2. ¿Cómo asumen el proceso de trabajo-retiro?}

El retiro laboral fue percibido como un hecho inevitable y generó una mezcla de emociones y vivencias en los participantes; para ellos, la transición implicó un cambio, una resignificación de roles y tareas construidas a partir del significado del trabajo y, a la vez, el enfrentamiento con una realidad: la partida.

Como parte de esta transición: la partida, entendida como la desvinculación y elaboración de una pérdida del rol laboral, los participantes comenzaron a construir sueños y se prepararon para la salida, a pesar de que el retiro laboral en la mediana edad no es parte de las tareas del desarrollo. Sin embargo, enfrentaron el retiro laboral como una tarea propia de sus vidas. Llama la atención la diferencia entre hombres y mujeres ante el retiro laboral.

"Yo presenté los papeles un diciembre, el último día que trabajamos, los presenté por presentarlos, porque ya me habían dicho que tenía el tiempo" (mujer No. 1).

Mientras que, para las mujeres, el retiro fue considerado como un hecho inevitable por tener que tomar la decisión de retirarse básicamente para no perder algunos beneficios. Los hombres, por su parte, tomaron la decisión en función del derecho que tienen y del deber cumplido. Se desprende, por lo tanto, que la jubilación es percibida como un mandato social.

"Cuando sale la resolución de que tengo derecho, yo mismo fui a los tribunales, recogí la resolución, la llevé a la Junta de Pensiones y pregunté: ¿dónde firmo?” (hombre No.1).

Asimismo, dejar de trabajar y acogerse a la pensión en una etapa de vida más temprana implicó una reevaluación de sus propios intereses, como parte de este proceso.

"Yo pienso que el deseo de cambiar de actividad, un poco más de libertad, y además que uno dice: "yo he sido muy feliz aquí, pero ya me toca el cambio»" (mujer No. 3)

Este modo de actuar coincide con lo señalado por la Teoría del Desarrollo Humano de que la mediana edad puede ser un tiempo de expectativas de género no cumplidas.

Por su parte, el trabajo remunerado les permitió a hombres y mujeres contar con los medios económicos para satisfacer las demandas personales, familiares, sociales, económicas y fue esencial en sus vidas para cumplir sus sueños y metas.

"El trabajo en mi vida, fundamental... Yo estaba totalmente identificada con el trabajo" (mujer No. 2). 
"Para mí, trabajar ha sido lo más importante, es un medio por el cual uno logra conocer amigos, mantener a la familia, darles una buena calidad de vida, el trabajo para uno es una buena opción, uno se vuelve útil" (hombre No. 3).

Para las mujeres el trabajo fue una fuente de realización y de poder al permitirles independizarse y concretar metas, valerse por sí mismas, ayudar a la familia y superarse para ser más competitivas.

"Para mí trabajar fue todo, fue liberarme en el buen sentido, ya no dependía de papi, y le pude dar más cosas a mi mamá, y en mi vida, en todo, yo hice todos los esfuerzos necesarios para no dejar de trabajar, porque mi esposo me insistía mucho en que dejara de trabajar" (mujer No. 1).

Desde el punto de vista de género y a partir de la inserción de la mujer en el mundo laboral la experiencia de adquirir poder económico acompañado por el hecho de generar recursos es lo que sostiene la tendencia actual de la mujer para prepararse para el trabajo y a subjetivarse como trabajadoras (Burin y Meler, 2001), lo cual pudo comprobarse en el caso de las mujeres participantes del estudio.

Al mantenerse como trabajadoras se aseguraron un lugar en el espacio público, les permitió tener una visión más amplia de su papel dentro de la sociedad.

“Trabajar en la U le ayuda a uno mucho, lo hace más abierto, entender un montón de cosas, yo, por ejemplo, me veo con mis hermanas, la forma de pensar, yo soy mucho más abierta que ellas. Uno aprende" (mujer No. 3).

De acuerdo con Murillo muchas mujeres no siempre aceptan cargos públicos que conlleven altas dosis de responsabilidad por el desgaste personal, lo cual afecta la demanda de espacio privado (Murillo, 1996). Es así como las mujeres entrevistadas señalaron estar conscientes de la limitación de su rol de madre, lo que influyó directamente en la toma de decisiones al dejar de lado su propia realización y la posibilidad de superarse y ocupar mejores puestos. Sin embargo, reconocieron que el trabajo fue gratificante tal y como lo expresaron dos de las participantes

"Gracias a Dios lo tuve, si bien es cierto yo me puse una pata encima, porque cuando existía la posibilidad de un puesto más alto en las oficinas, que implicaba la jornada de 
tiempo completo, siempre pensé que no valía la pena porque estaba mi hija de por medio, entonces fue una elección que yo hice" (mujer No. 3).

"El trabajo fue súper gratificante... siempre me gustó trabajar directamente con las personas... mi trabajo era muy administrativo, entonces me ponía a atender a los estudiantes, cosa que no me correspondía hacer, pero me encantaba” (mujer No. 2).

Lo expresado reafirma lo dicho por Murillo (1996); asimismo, para las mujeres, el trabajo tal y como lo exponen los teóricos tiene un significado especial, les permitió liberarse del mandato social de ser sólo madres y esposas y fue el medio para transitar sus roles de una forma más independiente a pesar de que este mandato tuvo gran peso en cuanto a las decisiones que tomaron, pues debieron sopesar su realización personal en el ámbito público con el mandato social. Por su parte, los hombres percibieron el trabajo como algo importante en sus vidas, pero lo asumen más como una tarea que tenían que cumplir.

"El trabajo para mí si fue importante, siempre quise algo mejor, la cuestión es que de pronto a uno lo absorbe la situación y entonces uno se queda ahí, pero siempre fue la idea mejorar y hacer lo mejor" (hombre No. 3).

La percepción de los hombres sobre el trabajo estuvo en función del medio que les permitió proveer a su familia de básico y no de su realización personal lo que concuerda con lo señalado por Gomaríz (1977) que desde el punto de vista del sistema patriarcal al hombre se le ha visto como el encargado de proporcionar los recursos necesarios para su familia, especialmente a sus hijos, lo que le ayuda en la conformación de su identidad genérica.

"Si yo no seguí estudiando más fue porque yo quise que ellos estudiaran... yo quería que todos mis recursos, mi esfuerzo y mi tiempo fuera calidad para ellos" (hombre No.1).

Además, la percepción de los hombres coincidió con lo planteado por Lagarde (1992), de que el hombre adulto ejerce pequeños poderes domésticos y familiares al tomar las decisiones en aspectos relacionados con el hogar, la educación de los hijos, los gastos familiares, entre otros.

En el proceso de despegue del trabajo hacia la condición de persona jubilada, la partida fue una realidad que no podían obviar al generar diferentes sentimientos y conductas. Volumen 11, Número 1, Año 2011, ISSN 1409-4703 
No había marcha atrás, estaban a las puertas de iniciar un nuevo estilo de vida. En general, los sentimientos de los hombres y las mujeres estuvieron asociados al retiro e íntimamente relacionados con el significado del trabajo. Entre los sentimientos experimentados estuvo el temor, la ansiedad, el optimismo, la angustia y el sentimiento de pérdida.

"No quería, tenía mucho susto, estaba contentísima trabajando, más bien me resistía a la pensión, yo decía: « ¿qué voy a hacer en la casa?, no, yo quiero estar trabajando» y me agarró como un pánico terrible... Empecé a pensar que iba a perder eso (derechos) yo no me quiero ir" (mujer No. 1).

Los sentimientos expresados estuvieron asociados con el significado del trabajo y con la construcción de su identidad. En el caso de las mujeres trabajadoras les brindó un estatus diferente a la mujer que no trabaja fuera del hogar, dado que la incorporación al mundo laboral le otorga poder al generar recursos que le permiten ser económicamente independiente (Lagarde, 1992 y Villarreal, 2005). De igual manera, la partida generó en las mujeres un sentimiento de pérdida, al ser el trabajo parte de su realización personal y ocupar un lugar dentro del espacio público.

"Sentí una gran pérdida, y decía: "jay no!, no voy a ir a la facultad, me voy a sentir mal», y cuando lo pensaba me daba cosa" (mujer No. 1).

En el caso de los hombres, si bien, el trabajo es un elemento constitutivo de la identidad masculina, se da una resignificación a partir del significado de la jubilación. Al asumir el nuevo rol, se toma el trabajo como un deber cumplido, un mandato social y la jubilación les permitió liberarse de este mandato social, y tener la posibilidad de seguir cumpliendo su rol proveedor por medio de la pensión. Unido a la partida y su significado está la pérdida de estatus y el sentido de pertenencia, aspectos que incidieron en el proceso de transición.

En el caso específico de una de las mujeres participantes, y la única que ocupó una posición de jefatura, dejar su rol laboral implicó la pérdida de estatus y de una condición de poder.

"El dejar de trabajar con un rol establecido es perder poder... era jefe y yo proyectaba mucho mandar. Obviamente, uno pierde como ese estatus, era aquella persona en función de eso" (mujer No. 2). 
El liderazgo genera un estatus laboral y social, la mujer entrevistada era reconocida por este rol y fue uno de los principales obstáculos que enfrentó en su proceso de ajuste a la jubilación, especialmente lo que ella llamó, pasar al anonimato.

Por su parte, el sentido de pertenencia que se desarrolla en una institución es fundamental, las personas participantes consideraron un privilegio trabajar en la Universidad de Costa Rica, institución cuya misión es la formación, al ser percibido como un espacio donde se formaban día a día y que les ayudó en su vida laboral, personal y familiar.

"La Universidad es una fábrica de personas, uno viene por acá no a sacar un título sino a ser mejores personas" (hombre No. 3).

"Para mí la universidad, fue una parte de mi vida y me siento muy orgulloso de haber laborado para la Universidad" (hombre No. 1).

De esta forma, se comprueba lo expresado desde el referente teórico, pertenecer a una organización laboral ayuda al sentido de pertenencia y al estatus laboral.

Como lo exponen algunos teóricos, el retiro laboral puede ocasionar una disminución de la autoestima y algún grado de depresión, señalándose que afecta más al hombre, por la subjetivización que hacen al visualizarse sólo desde el rol de trabajadores; sin embargo, en el caso del presente estudio son las mujeres las que, de alguna manera, expresaron sentir más esa pérdida de estatus.

De igual manera, un aspecto fundamental para enfrentar la transición del trabajo al retiro laboral radica en la madurez personal que permite tomar las acciones necesarias para enfrentar y sobrellevar la crisis que genera el cambio (Alvarez, 1995)

En el caso de la población en estudio, de las 6 personas participantes en la investigación cuatro de ellas consideraron oportuno asistir al Taller de Preparación a la Jubilación que imparte la Oficina de Recursos Humanos de la Universidad de Costa Rica. Este taller les ayudó para prepararse y asumir su nuevo rol.

"En el taller le abren a uno los ojos, le dicen a uno lo que va a pasar, ahí descubrí que sí, que era una opción para hacer cosas que nunca había podido hacer y he ido haciendo lo que quería hacer" (mujer No. 1).

"Llevé el curso, me abrió un poco de puertas, de expectativas para entender a lo que iba, descubrí que iba a otra cosa, ahí empezó mi jubilación" (hombre No. 2). 
Dos hombres manifestaron que tenían las herramientas y fortalezas para enfrentar este proceso.

"La verdad es que yo tomo las decisiones solo, sin contarle a nadie nada, creo que es una decisión personal" (hombre No. 3).

\section{3. ¿Cómo se visualizan como hombres y mujeres de la mediana edad en la condición de jubilados?}

Pasar del rol laboral al de persona jubilada da una resignificación de los roles y tareas que han desempeñado. Para la población en estudio, el cambio de estos roles coincidió con lo que denomina la crisis de la mediana edad. A raíz de esta crisis, los hombres y las mujeres tienen la sensación de que el tiempo se escapa, hay una conciencia más positiva, provocando la necesidad de atender sus propios intereses y pasatiempos, Gould citado por Rice (1997). De esta forma iniciaron un nuevo paso ¿qué podían hacer?, y ¿cómo podían ocupar su tiempo libre?

"Siempre les decía a las compañeras: "iqué lindo llevar algún día un curso de repostería, para aprender hacer algo!»” (mujer No. 1).

"Yo no terminé la $U$, y quiero volver a la U. Pienso llegar a ser antropólogo, tengo que empezar casi de cero, es algo que me gusta" (hombre No. 2).

Otro factor que interviene en el ajuste es la capacidad para la toma de decisiones, y para esto es importante clarificar lo que realmente quiere, si desligarse de toda actividad laboral o renovar una carrera con nuevas oportunidades laborales.

"Me volvieron a llamar a trabajar, y me fui a trabajar a la $U$, pero ya era diferente, porque era un lugar diferentísimo de donde yo había trabajado... Trabajé como por cuatro meses, fue muy bonita la experiencia, luego dije como que: “ya», eso me ayudó mucho" (mujer No. 1).

Como se puede apreciar, los y las participantes consideraron la posibilidad de explorar nuevos intereses, en función de su condición de persona jubilada, y no de la etapa de desarrollo en la que se encuentran. Esto concuerda con el planteamiento de Mata (2001) que uno de los factores que contribuye al ajuste a la jubilación es la capacidad que tiene la persona jubilada para explorar nuevos intereses

Volumen 11, Número 1, Año 2011, ISSN 1409-4703 
"Empecé a pensar: "jsí claro!», hay un montón de cosas que uno puede hacer y que había querido hacer toda una vida" (mujer No. 1).

Lo que nos lleva a que cuando los hombres y las mujeres han planeado de forma sensata y realista su retiro, lo afrontan de una manera más eficaz y optimista. Por último, en el proceso de despegue, un factor común fue la posibilidad de visualizarse como hombres y mujeres independientes, con capacidad de tomar decisiones, especialmente en lo económico.

Para las mujeres esto fue de gran importancia, pues en una sociedad patriarcal, como la nuestra, contar con una pensión les posibilita seguir manteniendo su autonomía.

"Mi vida familiar era muy importante, pero tampoco era sólo eso, entonces el trabajar medio día me ayudaba a tener mi actividad" (mujer No. 3).

"Yo estuve un tiempo trabajando medio tiempo porque él me decía, bueno es que yo vengo a almorzar y usted no está... yo hice todos los esfuerzos necesarios para no dejar de trabajar, porque mi esposo me insistía mucho en que dejara de trabajar" (mujer No. 1).

Desde su subjetividad este rol está siempre presente, pero con una connotación diferente, sienten libertad para realizar otras actividades, lo que implica que se da una resignificación de los roles desempeñados.

Al interiorizar su rol de persona jubilada les permite reevaluar los roles que hasta el momento han desempeñado, y acogerse a la pensión implica haber cumplido con ese mandato social, y en este momento se dan permiso para disfrutar de "su momento".

\section{4 ¿Cómo se visualizan en su condición de jubilados?}

La partida y sus implicaciones fue trascendental en el proceso de ajuste, pero haber concretado sueños y expectativas durante la transición del trabajo al retiro laboral fue fundamental, así como contar con el apoyo de la familia.

Dejar de trabajar les permitió más tiempo para realizar otras actividades, dedicarse a la familia, disfrutar y tener más paz y tranquilidad. 
“La pensión significó una liberación, descubrí que sí, que era una opción para hacer cosas que nunca había podido hacer y he ido haciendo lo que quería hacer" (mujer No.1).

"Fue como una liberación, más bien que me dieron permiso de hacer todo eso" (mujer No. 3).

El retiro laboral significó libertad, darse permiso para realizar actividades que desde el punto de vista social no tiene el mismo valor que el trabajo, como la recreación y el disfrute; en ese sentido, el retiro laboral en la mediana edad puede tener a nivel social, una connotación negativa. La persona trabajadora aún puede dar mucho.

"Es casi la comidilla de cuando le preguntan a uno: “¿qué estás haciendo?... Mirá, estoy pensionado. ¡Cómo! No seas vagabundo»” (hombre No. 2).

A pesar de las críticas, no perciben pensionarse como algo negativo, lo consideran un derecho, lo que les permitió enfrentar el proceso de manera más positiva y concretar las expectativas que tenían cuando iniciaron el proceso de transición fue esencial para el ajuste. En la vivencia y ajuste al rol de persona jubilada, un aspecto fundamental es el cambio en el estilo de vida.

En el caso de las personas que se jubilan en una etapa de vida más temprana, el cambio del estilo de vida tiene que ver con la reorganización del tiempo libre, y con cambios propios de la etapa de vida, que los asumen de manera positiva, sin ser un impedimento para tener un estilo de vida más saludable; al contrario, se cuidan más que antes. Otro aspecto fundamental fue la reestructuración del tiempo, pasar del tiempo laboral al tiempo subjetivo.

"Mi rutina cambió completamente, no es lo mismo levantarse para cumplir con un horario, con un trabajo, a levantarse para hacer lo que uno desea" (hombre No. 3).

Las personas entrevistadas coincidieron que el cambio de la rutina y la reestructuración del tiempo no fue problema. Pareciera que fue manejable a raíz de la información que obtuvieron del taller de preparación a la jubilación. 
Es importante señalar que algunos de los entrevistados, tanto hombres como mujeres, señalaron que los primeros meses de la jubilación se sintieron de vacaciones, no fue hasta después que empezaron a ocupar el tiempo libre.

"Los primeros tres meses yo sentí que eran vacaciones" (hombre No. 2).

"Yo me pensionaba el 1 de enero, y entonces para mí fue lindísimo, ahora me voy sentir como que no estoy pensionada" (mujer No. 1).

Esta percepción coincide con la fase de euforia de la jubilación. Se vive un bienestar, una alegría al verse sin la obligación de ir a trabajar.

Los participantes ocupan su tiempo en diferentes actividades, lo que se relaciona con el concepto de ocio, pues el ocio son todas aquellas cosas u ocupaciones que se hacen de manera voluntaria para descansar, divertirse o bien para dedicarse a la participación social voluntaria (Dumazedier, 1971)

"Yo quiero disfrutar mi vida de otra manera, yo no me veo estudiando, no me veo sacando una carrera, trato de disfrutar el momento, el segundo a segundo, salir, disfrutar" (hombre No. 1).

Desde el punto de vista psicológico se da una resignificación al estilo de vida para tratar de mantenerse tan bien y saludable como sea posible (Rice, 1997 y Le Shan citado por Bonilla, 1983).

Otro aspecto en la vivencia y ajuste a la jubilación son las pérdidas asociadas al rol laboral. Para dos de las mujeres, dejar su rol laboral fue una pérdida, el trabajo implicó realización personal.

"Ahora yo voy a ser "J", no voy a ser ni la jefe de... ni la que le resuelve el problema a todo el mundo, ni la que todos Ilaman para todo, porque yo era en función del trabajo, yo era una persona conocida en función del trabajo... me tuve que trabajar mucho en la autoestima porque después de tener un perfil muy alto por el trabajo y por el puesto, de pronto usted pasa al anonimato" (mujer No. 2).

Por su parte, los hombres, consideraron que al dejar su rol laboral enfrentaron una pérdida en el contacto social y ahora se circunscribe más a la familia. 
"Tengo menos relaciones sociales con las personas, la relación sociales que tengo son casi con la mayoría con las personas que convivo, mis padres, los vecinos, la familia" (hombre No. 1).

Sin embargo, las mujeres entrevistadas no sienten que el retiro laboral haya implicado un cambio en su rol social, porque se mantienen en contacto con sus compañeros y compañeras de trabajo y también porque las actividades que realizan les han permitido crear nuevas relaciones.

"Me llaman que tienen diferentes actividades y yo voy y me siento bien" (mujer No. 1).

Para el proceso de ajuste y adaptación al nuevo rol es importante el apoyo que reciba de su familia, especialmente de sus hijos y su pareja. En el caso de las personas participantes del estudio, solo uno de los entrevistados ha tenido ciertos roces que la familia y los hijos.

"El caso de mi esposa y de mis hijos, no estaban preparados para verme jubilado, entonces, uno que tiene otras obligaciones y estas cambian, quieren que asuman otras obligaciones... la compañera de uno no ha entendido, bueno en su momento entenderá, igual con los hijos de uno" (hombre No. 3).

Otro factor que incide en el proceso de ajuste son los roles y estereotipos que se manejan a nivel social. Se considera que el rol laboral, ser productivo, hace a la persona trabajadora sentirse útil y ocupar un lugar en el espacio público.

Dejar el rol laboral en una etapa de vida considerada como la más productiva puede ser percibido a nivel social de forma diferente, si quien se jubila es hombre o mujer. En el caso de las mujeres se considera, incluso por los mismos hombres, que para ellas es más fácil la adaptación al nuevo rol, porque la mujer está más acostumbrada a la doble jornada.

"Yo siento que puede ser más fácil para la mujer, por el rol que lleva, la mujer está acostumbrada a dos trabajos" (hombre No. 1).

De igual forma, la percepción que tienen las mujeres es que para el hombre es un poco más difícil dejar su rol laboral.

"Muchos hombres no se quieren pensionar, porque dicen que qué van hacer en la casa. En los hombres yo sí pienso que es más difícil” (mujer No. 1). 
Lo anterior nos lleva, una vez más, a la diferenciación del rol con respecto al género. El rol de la mujer está más en función del mundo privado, lo que supone una mejor adaptación que el hombre. Sin embargo, a nivel personal tanto hombres como mujeres se visualizan en forma diferente.

"En el contexto social hay un mandato: "ahora que te pensionés qué dicha para que estés en tu casa...» Hay una presión social, por ejemplo, que hay que chinear al marido, para que haga cosas en la casa o para que esté en la casa...Yo sí siento que hay una visión de que la mujer se pensiona para que vaya hacer oficio, o para que esté en la casa o que cuide nietos, y si usted no tiene hijos, entonces usted va a ser una vagabunda" (mujer No. 2).

Otro aspecto es la percepción que se tiene a nivel social de la persona jubilada en una etapa de vida más temprana. En el caso de las personas entrevistadas, éstas coincidieron que socialmente no es bien visto que una persona joven deje de trabajar.

"La gente lo ve como una carabarrada: "jmae!, no seas tan cara de barro», me dicen amigos y amigas. Cuando ven que uno se ha pensionado, se molestan. Tan joven, en general a la gente le molesta ver a una persona joven y más cuando no se ve más joven, que no aparenta, entonces les parece que no, que pueden producir y dar más" (hombre No. 2).

Todos concuerdan que a nivel social no se ve con buenos ojos que una persona joven se acoja a la pensión, pero no les causa malestar, porque lo consideran un derecho que se han ganado.

\section{Conclusiones}

En este apartado, se exponen las conclusiones. La revisión bibliográfica, que sirve como referente teórico, brindó los aportes conceptuales para dar respuesta a la pregunta y subpreguntas de investigación.

La información brindada por las personas entrevistadas ayudó a apoyar o refutar dichos supuestos teóricos y las conclusiones se dan a partir del análisis fenomenológico que permitió comprender lo concebido y subjetivo de la vivencia del proceso de transición y el ajuste a la jubilación. 
Se buscó interpretar el sentido individual a partir de las creencias y convicciones que para cada uno de los participantes son reales, pues tal y como lo señala Schütz (1974) cada ser humano percibe las cosas desde una óptica diferente, al tener como referente su realidad social.

Las conclusiones se dan en un marco de una concepción dialéctica, desde lo subjetivo, por un lado, que implicó la historia de vida y desde el mandato social, por otro, por lo roles asignados y asumidos por la población en estudio.

- Por los roles asignados y asumidos, la experiencia de vida, la transición y ajuste a la jubilación tienen un significado diferente según la condición de género.

- El factor económico fue el que más influyó en la decisión de acogerse a la pensión.

- Desde la condición de género, las mujeres consideraron la posibilidad de continuar su rol laboral, lo que contradice lo estipulado teóricamente de que el rol de madre y esposa es lo que predomina.

- Para los hombres, el trabajo no fue fundamental, el cambio de rol implicó la posibilidad de dedicarse al ocio y nuevos intereses.

- El retiro laboral es asumido a partir de la experiencia de vida, del significado del trabajo.

- Para las mujeres, el trabajo fue percibido como una transformación de los roles socialmente asignados, lo que les da un reconocimiento y estatus en el espacio público.

- Por el significado del trabajo, las pérdidas asociadas al retiro tienen más peso en las mujeres.

- El trabajo como fuente de identidad y de estatus no tiene mayores implicaciones para los hombres.

- Hombres y mujeres asumen la jubilación y sus implicaciones como algo natural.

- La exploración de intereses no fue desde lo vocacional, sino para su realización personal.

- La percepción de sí mismo está en función de lo sujetivo, de la vivencia del nuevo rol y no de la etapa de vida.

- Al asumir la pensión cierran una etapa de vida y se da una resignificación de los roles.

- Se reconstruye la identidad desde lo subjetivo al asumir la condición de persona jubilada. 
- Existe una relación inversa. Los hombres toman las decisiones en forma directa, mientras que las mujeres parece ser que se da un continuo, pues se mantienen desempeñando el rol de madres y esposas.

- Los ajustes personales y sociales están en función de su vivencia, del momento histórico, de los roles y del apoyo familiar.

- Este grupo ha vivido, desde su vivencia subjetiva y al momento del estudio las fases de euforia, ocupación y luna de miel.

- La jubilación implica una resignificación de la identidad de género. Permite a los hombres mantener el rol de proveedores y protectores y a las mujeres el de madres y esposas, pero con independencia económica, de acuerdo con el ingreso de la pensión.

\section{Recomendaciones}

Al finalizar este estudio surgen una serie de recomendaciones para el abordaje del proceso de transición del trabajo al retiro laboral y el ajuste a la jubilación en personas que se encuentran en la etapa de la mediana edad.

Se plantean a continuación las siguientes recomendaciones que pretenden ampliar los conocimientos desde la disciplina de la Orientación, del proceso de ajuste a la jubilación:

- Se recomienda realizar nuevas investigaciones que permitan comparar las fases de la jubilación en personas recién jubiladas y otras que tengan tres o cuatro años de jubilados para ampliar los conocimientos desde la disciplina de la Orientación del proceso vivencia y ajuste a la jubilación.

- Se recomienda realizar nuevas investigaciones sobre la vivencia de la jubilación en las diferentes etapas del ciclo vital para explorar cómo se vive el retiro laboral.

- Se recomienda a las instancias universitarias acoger los resultados de este estudio para enriquecer el proceso de orientación de los funcionarios y las funcionarias próximas a retirarse para que se preparen a nivel personal, familiar y social de manera tal, que la transición y ajuste sea lo más favorable posible.

- Para la disciplina de la Orientación, en los procesos de transición y ajuste a la jubilación tener presente la condición de género, la construcción de la identidad y el significado del trabajo. 
- Que la Universidad de Costa Rica mantenga espacios que permitan a las personas jubiladas mantenerse vinculadas a la institución, por medio del aporte de su experiencia y conocimiento en comisiones, investigación y como instructores.

\section{Referencias}

Abarca, Sonia y León, Moisés. (1990) La jubilación: conceptos básicos. San José, Costa Rica: CENDEISSS, Editorial Nacional de Salud Seguridad Social.

Alvarez, Manuel. (1995). Orientación Profesional. Barcelona, España: CEDECS.

Amén, Rosita. (2002). Comparta y Disfrute la jubilación. Tesis de grado para obtener el título de Master en Gerontología. Sistemas de Estudios de Posgrado, Universidad de Costa Rica, San José, Costa Rica.

Bonilla, Amparo. (1998). Los roles de género. Madrid: Ediciones Pirámide, S. A.

Bonilla, Flory Stella. (1983). Crisis de la vida del Adulto. Material mimeografiado del curso de Orientación del Adulto, de la Carrera de Orientación, UCR.

Buendía, Leonor y colaboradores. (1998). Métodos de investigación en psicopedagogía. España: Ediciones Mc Graw Hill.

Burin, Marcela y Meler, Irene. (2001). Género y Familia. Poder, amor y sexualidad en la construcción de la subjetividad. Bueno Aires: Editorial Paidós.

Campos, Alfredo. (1984). Orientación no Directiva. Barcelona, España: Editorial Herder:

Carballo, Nora. (1994). Aportes del paradigma sistémico a las diferencias de género en el Ciclo Vital Familiar. Revista Prospectiva (1).

Castrillón, Marcela. (1995). Impacto Psicosocial de la Jubilación. Tesis sometida a la consideración de la comisión del Programa de Estudios de Postrado en Psicología para optar al grado de Magíster Scientice en Psicología. Universidad de Costa Rica, San José, Costa Rica.

Centro Centroamericano de Población, Universidad de Costa Rica. (2006). Costa Rica: Estimaciones y proyecciones de población 1970-2100 actualizadas al 2000. Disponible en: http://ccp.ucr.ac.cr/observa/CRnacional/proyeccion.htm

Cerda, Hugo. (1991). Los elementos de la investigación. Bogotá, Colombia: Editorial CODICE.

Dietrich, Georg. (1986). Psicología General del Counseling. Barcelona, España: Editorial Herder. 
Dumazedier, Joffre, (1971). Realidades del Ocio. En Ideología en Ocio y sociedad de clases. Barcelona: Editorial Fontanella S. A

Fandiño, Dyalá. (2000). Jubilación: Cambios en las Relaciones Sociales. Revista Anales en Gerontología, 2 (2), 31-42. San José, Costa Rica.

Fernández, Lourdes. (2000). Roles de género y mujeres académicas. Revista de Ciencias Sociales, 42 (88), 63-75. San José, Costa Rica.

Flick, Uwe. (2004). Introducción a la Investigación cualitativa (Traducido por Tomás del Amo). Madrid. España: Ediciones Morata, S.L.

Gomáriz, Enrique. (1997). Introducción a los Estudios sobre masculinidad. San José: Centro Nacional para el Desarrollo de la Mujer y la Familia.

Gordillo, María Victoria. (1988). Manual de Orientación Educativa. Madrid: Ediciones Morata.

Hermida, José Manuel. (2006). Del trabajo al trabajo...Orientaciones hacia el trabajo remunerado de las mujeres en Costa Rica. San José, Costa Rica: UNIFEM.

Hernández, Roberto y otros. (2000). Metodología de la Investigación. México: Mc GrawHill.

Instituto Nacional de Estadísticas y Censo. (2005). Censo de población 2005. San José: INEC. Disponible en www.inec.go.cr

Lagarde, Marcela. (1992). Identidad de Género. Curso en el Centro Juvenil "Olof Palme". Managua, Nicaragua.

Lagarde, Marcela. (1994). Género en el desarrollo. Revista Horizontes Cooperativos, (6), 56-63. San José, Costa Rica.

Lagarde, Marcela. (1995). Cautiverio de las mujeres: madre-esposas-monjas, putas, presas y locas. México: Universidad Nacional Autónoma de México.

Mata, Alejandrina. (1993). Identificación de los Factores Personales y Ambientales que facilitan el ajuste de la persona a la jubilación. Tesis de grado para obtener el título de Master en Gerontología. Sistemas de Estudios de Postgrado, Universidad de Costa Rica, San José, Costa Rica.

Mata, Alejandrina. (2001). Algunos Factores que facilitan el ajuste de la persona a la jubilación. Revista Anales en Gerontología, 3 (3), 27-34, San José, Costa Rica.

Millet, Kate. (1995) Política sexual. Cátedra, Madrid: Instituto de la Mujer. Disponible en www.europrofem.org

Moore, Henrietta. (1991) Antropología Y Feminismo. Madrid, España: Ediciones Cátedra 
Murillo, Soledad. (1996). El Mito de la Vida Privada, De la entrega al tiempo propio. España: Siglo XXI de España Editories, S.A.

Peiró José Manuel y Prieto Fernando. (1998). Tratado de Psicología del Trabajo (Vol. II, Aspectos sicosociales del trabajo). España: Editorial Síntesis Psicología.

Pereira, María Teresa. (1998). Orientación Educativa. San José, C.R.: Editorial EUNED.

Ramón Gina y Fonseca, Yalile. (1997). Factores Sociales que Inciden en el Sistema Familiar como Producto de la Jubilación, Tesis presentada para optar por el grado de Licenciatura en Trabajo Social, Universidad de Costa Rica. San José, Costa Rica.

Rice, Philip. (1997). Desarrollo Humano, Estudio del Ciclo Vital. México: Prentice-Hall Hispanoamericana S.A.

Rivas, Francisco. (1995). Manual de Asesoramiento y Orientación Vocacional. Madrid: Síntesis Editorial.

Rodríguez Gregorio, Gil, Javier, y García, Eduardo. (1996). Metodología de la Investigación Cualitativa. Málaga: Ediciones ALJIBE.

Rodríguez, María Luisa. (1992). El mundo del trabajo y las funciones del orientador. Propuestas formativas. Barcelona: Editorial Ariel, S.A.

Roger, Carl. (1984) Orientación Psicológica y Psicoterapia. Madrid, España: Narcea

Schütz, Alfred. (1974). El problema de La realidad social. Buenos Aires, Argentina: Amorrortu.

Strauss, Levy y Cardenas, José. (1988). El significado del trabajo: necesidades y satisfacciones del trabajador. En Personal, problemas humanos de la Administración. México: Prentice-Hall Hispanoamericana, S.A.

Taylor, Steve, Bogdan, Robert. (1998). Introducción a los métodos cualitativos de investigación (Traducido por Jorge Piatigorsky). España: Ediciones Piadós Ibérica, S.A.

Valles, Miguel. (2003). Técnicas cualitativas de Investigación Social. Reflexión metodológica y práctica profesional. Madrid, España: Proyecto Editorial SíNTESIS.

Villarreal, Cecilia. (1996). La Menopausia: un proceso de crecimiento. Tesis sometida a la consideración de la Comisión del Programa de Estudios de Posgrado en Estudios de la Mujer para optar al grado de Magíster Scientae. Universidad de Costa Rica, Universidad Nacional. San José, Costa Rica.

Villarreal, Cecilia. (2005). Mujeres en la mediana edad. Desafíos y replanteamientos vitales durante la menopausia. San José: Editorial Fundación UNA.

Weber, Max. (1969). Economía y Sociedad. Esbozo de sociología comprensiva. México: FCE. 